noch nicht im Detail erörtert worden seien. Der Dispositionsrahmen beim Brückenschlag des Vermittlungsausschusses wird demzufolge wohl immer wieder neu zu vermessen sein und dies in Abhängigkeit der wechselnden unterschiedlichen parteipolitischen Mehrheiten in Bundestag und Bundesrat, die der Autor abschließend aufzeigt.

Mehrere Autoren kommen in ihren Beiträgen auf das zuletzt im Jahre 2008 vom Bundesministerium der Justiz herausgegebenen Handbuch der Rechtsförmlichkeit zu sprechen. So wendet sich die dortige Referatsleiterin Dorothee Weckerling-Wilhelm Anforderungen der Rechtsförmlichkeit zu und schlägt damit ein Kapitel auf, das die entscheidende Bedeutung formaler Erfordernisse beim Zustandekommen materiellen Rechts herausstellt. Seit Geltungsbeginn des Grundgesetzes im Jahre 1948 gehe es beim Erlass von Gesetzen und Verordnungen um rechtliche Unangreifbarkeit und rechtsförmliche Einheitlichkeit der Bundesgesetzgebung, erinnert die Autorin und erläutert, worauf aus ihrer fachlichen Sicht zu achten sei, wenn politische Entscheidungen durch ein konkretes Gesetzesvorhaben umgesetzt werden sollen: Ziel sei dabei „die Schaffung und der Erhalt eines in sich stimmigen Normbestands“.

Wie dieser Normbestand zustande kommt, abgeändert, zurückgenommen und erweitert wird und wie er so im Wandel besteht - das zeigen beide Bücher vortrefflich auf, öffnen und fördern das rechtshistorische und theoretische Wissen um die Gesetzgebung und vor allem das praxisorientierte Verständnis für sie.

Friedemann Ey

\title{
Praktischen Widerständen und theoretischen Erwartungen zum Trotz: Steuern, Regieren und Exekutivhandeln moderner politischer Systeme
}

Dahlström, Carl, B. Guy Peters und Jon Pierre (Hrsg): Steering from the Centre: Strengthening Political Control in Western Democracies, Toronto University Press, Toronto 2011, 288 Seiten, $\$ 27,95$.

Falkner, Gerda (Hrsg): The EU's Decision Traps. Comparing Policies, Oxford University Press, Oxford 2011, 260 Seiten, £ 57,-.

Lodge, Martin und Kai Wegrich (Hrsg): Executive Politics in Times of Crisis, Palgrave Macmillan, Basingstoke / Houndsmills 2012, 312 Seiten, £ 63,-.

Politik und Regieren werden ebenso komplexer wie diffuser. Dies stellt zwar kein Novum dar, bedenkt man, dass mit der Entwicklung und Modernisierung von Staatlichkeit und Politik generell auch Prozesse der funktionalen Ausdifferenzierung und institutionellen Spezialisierung einhergingen. Die neuere Vergangenheit in modernen politischen Systemen scheint aber besonders von wachsender Komplexität der Regierungswirklichkeit mit zunehmender politischer Dispersion gekennzeichnet zu sein. Festgemacht wird dies in der Politikwissenschaft zum einen an Entwicklungen wie der Globalisierung, Transnationalisierung, Europäisierung und der allgemeinen Entgrenzung von Problemlagen. Von fundamentaler Bedeutung in diesem Kontext gelten zum anderen die hierauf reagierenden Regulierungs- und Problembewältigungsansätze, die entsprechend grenzübergreifende Arrangements zur Kooperation, Koordinierung und Konsensfindung benötigen. Aber auch innerhalb politischer Systeme treten parallele Auflösungs- und Diversifizierungserscheinun- 
gen hervor, beispielsweise angesichts von Regionalisierungstendenzen oder des Wandels in gesellschaftspolitischen Werten, Identitäten oder den sich häufig widersprechenden Erwartungen, die an ein demokratisches, legitimes und effektives Regieren gestellt werden. Ebendiese gehören zur Konstellation an Grundzügen und entsprechenden Forschungsfragen zum Wandel von Regieren und Staatlichkeit.

Sicherlich verwundert es nicht, dass ein solcher Themenkomplex zu einem gewissen Defätismus bezüglich der Regierungs- und Steuerungsfähigkeit der Politik einladen kann. Auch die hier zu besprechenden Bücher problematisieren vor dem oben skizzierten Hintergrund ihre Forschungsgegenstände und weisen auf verschiedene Defizite - ob performativer oder legitimatorischer Art - hin. Allerdings haben diese Studien ebenfalls gemeinsam, dass sie sich insbesondere mit Problemlösungsansätzen zur kollektiven Entscheidungsfindung im Zeichen komplexen Regierens in mehreren Kontexten befassen. Es handelt sich bei ihnen also um Auseinandersetzungen mit den Herausforderungen gegenwärtiger Politik und um differenzierte Analysen der mitunter erfolgreichen Anpassungen, mit denen die Handlungskapazität politischer Systeme aufrecht erhalten beziehungsweise herbeigeführt werden soll. Sie überprüfen in mehreren Fallstudien gepaart mit vergleichenden Perspektiven, wie und inwiefern Regierungssysteme beziehungsweise bestimmte Regierungsinstitutionen über Delegation und Deregulierung hinaus eigene Regulierungs- und Steuerungsfähigkeit (wieder) erlangen. Als übergreifendes Forschungsinteresse werden dabei sowohl strukturell-institutionelle als auch politikfeldspezifische Faktoren der jeweils betrachteten Entscheidungssysteme berücksichtigt. In methodologischer Hinsicht weist jeder Band zudem einen systematischen Pluralismus auf, indem durchgängig qualitative und quantitative Ansätze sowie die Makroperspektive mit tiefergehenden Fallbeispielen kombiniert werden.

Trotz erheblicher Überschneidungen unterscheiden sich die Bände dennoch grundlegend in Bezug auf die jeweilige Schwerpunktsetzung und damit verbundene Fallauswahl, wodurch die gemeinsame Lektüre ein besonders erkenntnisreiches Unterfangen wird. Während „The EU's Decision Traps“ hauptsächlich die europäische Ebene der Entscheidungsfindung anhand einzelner Politikfelder zum Gegenstand macht, werden in „Steering from the Centre“ westliche Länderbeispiele aus Europa, Nordamerika und Australien untersucht; „Executive Politics in Times of Crisis" vereint hingegen sowohl themen- und policy-orientierte als auch länderspezifische Studien. Angesichts des Umfangs der drei Bände, kann im Folgenden nur versucht werden, zumindest einen Überblick über die Themen und theoriebildenden Analysen zu verschaffen. Gerda Falkner versammelt eine Reihe von EU-Experten, welche die bisher entwickelten Auswege aus der Politikverflechtungs- und Entscheidungsfalle in einzelnen Politikbereichen aufspüren. Untersucht werden nach dem Einleitungskapitel der Herausgeberin, das einen Analyserahmen für die weiteren Beiträge absteckt, klassische, für Entscheidungsblockaden anfällige Politikfelder wie die Gemeinsame Agrarpolitik (Christilla Roederer-Rynning), Binnenmarkt (Susanne Schmidt), Finanzmarktregulierung (Zdenek Kudrna), Justiz und Inneres (Florian Trauner), Gemeinsame Außen- und Sicherheitspolitik (Nicole Alecu de Flers / Laura Chappell / Patrick Müller) und Sozialpolitik (Dorte Sindbjerg Martinsen / Gerda Falkner), aber auch neuere beziehungsweise bisher oft vernachlässigte EU-Politiken wie Steuerpolitik (Philipp Genschel), Energiepolitik (Johannes Pollak / Peter Slominski) und Umweltpolitik (Katharina Holzinger). Hinzu kommen Querschnittsbeiträge zu politikfeldübergreifender Koordinierung (Miriam Hartlapp), ein Vergleich zwischen nationaler und supranationaler Erfahrung in der Bewältigung von Entscheidungsfallen insbesondere am Beispiel der Verfassungspolitik (Arthur Benz), ein Resümee mit einer Konzepter- 
weiterung durch den „Urheber“ der Politikverflechtungsthese (Fritz W. Scharpf) sowie ein abschließendes Vergleichskapitel zu den Ergebnissen und generalisierbaren Themen (Gerda Falkner).

Herausgearbeitet werden somit zum einen die Dilemmata, die sich aus dem - auch demokratietheoretisch relevanten - Spannungsverhältnis zwischen grenzübergreifendem Koordinierungs- und Regulierungsbedarf einerseits und der Aufrechterhaltung von staatlicher Eigenständigkeit und der Autonomieschonung andererseits ergeben. Zum anderen legen die Studien dar, dass entgegen einigen üblichen Theorieannahmen und trotz Verflechtungshürden eine Reihe von Prozessen und Strukturen dazu beitragen, dass die EU und ihre Mitgliedstaaten, zum Teil mit erstaunlicher Effektivität, der „Entscheidungsfalle“ entkommen. Für viele Politikfelder spielten diverse „Schatten“ eine wesentliche Rolle, beispielsweise Gesetzgebung im Schatten der (qualifizierten) Mehrheit im Rat oder die Entscheidungsfindung im Schatten eines durch die Judikatur des Europäischen Gerichtshofes veränderten Status Quo, was wiederum die Kommission bei der Agenda-Setzung und Gestaltung ihrer Regulierungsvorstöße zu nutzen wusste (zum Beispiel Binnenmarkt). Ein weiteres Phänomen, das auch eine große Rolle in den Studien zu „Steering from the Centre“ und „Executive Politics in Crisis“ spielt, zeigt sich in den Maßnahmen zur institutionellen Weiterentwicklung und „Arenen-Verschiebung“ („arena shifting“). Solche Steuerungs- und Problemlösungsstrategien bestehen etwa darin, dass bestimmte Sachfragen auf andere, oft neu errichtete Gremien verlagert werden (siehe insbesondere die Beiträge von Benz, Falkner und Kudrna). Als paralleles Dilemma wird jedoch deutlich, dass erfolgreiche Auswege aus den EU-Entscheidungsfallen häufig nicht-intendierte Negativfolgen und Verluste bei anderen hohen Gütern mit sich bringen. So treten zwickmühlenartige Situationen auf, in denen den Mitgliedstaaten der eigenständige Handlungsspielraum in einem Politikbereich geraubt wird (zum Beispiel in der Steuerpolitik), während bei der Konsensfindung eine suboptimale Entscheidung akzeptiert wird, die mehrfache Ausnahmen, Ausweichoptionen und Ähnliches beinhalten kann (zum Beispiel Umweltpolitik) oder Marktschaffung über Marktkorrektur asymmetrisch verfolgt (wie in der Sozialpolitik besonders ersichtlich).

Analog zu den obigen politikfeldvergleichenden Studien untersucht „Executive Politics in Times of Crisis“ Wandel, Reformen und Handlungsmöglichkeiten exekutiver Politik. Dies geschieht zum einen vor dem allgemeineren Hintergrund struktureller und funktionaler Herausforderungen modernen Regierens, was im ersten Teil über die generellen Themen und Entwicklungstendenzen behandelt wird. Anlass für den Band waren zum anderen neuerliche Krisen und die darauf folgenden Reaktionen, die im zweiten Teil behandelt werden. Im Einleitungskapitel (Martin Lodge und Kai Wegrich) erläutern die Herausgeber, die auch einen Beitrag zu Politikinstrumenten und Exekutivpolitik sowie ein Gesamtschlusskapitel verfassen, die Bewegründe, Vorgehensweise und Struktur des Bandes. Insgesamt wird dabei angestrebt, die Forschung über den Konnex zwischen vergleichender Regierungslehre und Verwaltungswissenschaft weiter zu entwickeln. Daran anknüpfend erfolgt im ersten Teil die Auseinandersetzung mit übergreifenden Entwicklungen im Verhältnis zwischen den administrativen und politischen Dimensionen der Exekutivpolitik sowie der Politik als Governance und als Policy. Hierzu gehören die Beiträge über die Auswirkungen der Internationalisierung einzelstaatlicher Bürokratien (Kutsal Yesilkagit), präsidentielle Interventionen zur Politisierung der Exekutivgewalt im in den USA (David Lewis), über die Ausbreitung betriebswirtschaftlich-orientierter Reformen wie New Public Management in westlichen Demokratien (Philippe Bezes) sowie in den post-kommunistischen Staaten Mittel- und Osteu- 
ropas (Jan-Hinrik Meyer-Sahling / Will Lowe / Christian van Stolk), über Haushalts- und öffentliche Finanzpolitik als Teil der Exekutivpolitik (Philipp Krause), Konzeptualisierungen der Entwicklungshilfe zur Verwaltungsmodernisierung in Entwicklungsländern (Nilima Gulrajani) sowie über die Responsivität von Regulierungsbehörden mit einer Fallstudie zur britischen Finanzdienstleistungsaufsicht (Sharon Gilad).

Darauf folgen im zweiten Teil Analysen über Krisenmanagement und Koordinierungsmaßnahmen durch die Exekutive anhand mehrerer Beispiele in unterschiedlichen Ländern (Arjen Boin / Paul 't Hart), exekutive Arbeitsweisen und die Zentralisierung der Kernexekutive insbesondere im Kontext der Finanzkrise und der Kreditklemme (Salvador Parrado), über die Verknüpfungen zwischen Krisen, Governance und exekutiver Handlungskapazität (Felicity Matthews), das Zwischenspiel zwischen administrativer Planung und exekutiver Politik im Kontext von Großprojekten und deren Leistungsdefiziten samt Fallbeispielen aus UK, Kanada, Frankreich, Australien und den USA (Will Jennings), und schließlich ministerienübergreifende Koordinierung in der Klimapolitik am Beispiel Deutschlands (Julia Fleischer / Thurid Hustedt). Diese Studien, die nicht nur ein breites Spektrum an Themen, sondern auch zahlreiche Länderbeispiele erfassen, schaffen verstärkte Klarheit zu einer Reihe von Entwicklungstendenzen; zusammengenommen ergeben sie ein eher ambivalentes und somit doch zutreffendes - Bild gegenwärtiger Politik. So scheinen mehrere Dualismen und entgegengesetzte Strömungen aufeinander zu treffen. Dies zeigt sich wohl nirgendwo deutlicher als im Kontext der Krise. Gerade dann werden in der Regel kurzfristige Entscheidungen und konzertierte Handlungen seitens der Exekutive gefordert, die jedoch aufgrund längerfristiger Tendenzen der strukturellen und funktionalen Streuung ihrer Gewalt (neu) gestärkt werden muss, denn ihre vorherige Schwächung ermöglichte häufig erst die Entstehung der Krise. Damit hängen weitere Spannungsfelder der Exekutivpolitik zusammen - von Transnationalisierung und Entgrenzung gegenüber Renationalisierung bis hin zu zunehmender gesellschaftlicher Fragmentierung und Ausdifferenzierung gegenüber steigendem Koordinierungsbedarf. Darüber hinaus wird noch grundlegender die enorme politische wie politikwissenschaftliche Bedeutung der Exekutive untermauert.

Ein Dualismus zwischen langfristigen Entwicklungen und neueren Reformen stellt auch den Hauptausgangspunkt für den Band „Steering from the Centre. Strengthening Political Control in Western Democracies“ dar. Hier geht es weniger um Auslagerung- und Diffusionsprozesse - von Dezentralisierung, Devolution und Delegation zur Deregulierung -, die die Herausgeber (Carl Dahlström / B. Guy Peters / Jon Pierre) unter dem Begriff „Entzentrieren“ („decentring“) subsumieren, sondern um Entwicklungen und Maßnahmen, die zur Stärkung der Steuerungsmöglichkeiten oder „Rezentrierung“ führen. Angesiedelt sind die Beiträge primär an der Kernexekutive (Premierminister und Präsidenten im Falle Frankreichs und der USA) der jeweils behandelten Systeme. Im Einleitungskapitel der Herausgeber wird eine Trias von Ansätzen vorgestellt, an denen sich die weiteren Fallstudien orientieren. Im Gegensatz zur Aufgabe („Letting go“) von Zuständigkeiten richten sie eher den Blick auf die beiden Strategien zur Stärkung der Steuerungskapazität vom „Zentrum“ aus. Diese nennen sie Festhalten („Holding on“) - zum Beispiel Politisierung der Exekutive mit entsprechenden Rekrutierungsmaßnahmen - und Wiederherstellung („Restoring the centre“) - das heißt vor allem Ausbau der unmittelbaren Koordinierungsmöglichkeiten des Regierungschefs. Untergliedert wird der Band entlang vier Ländergruppen unterschiedlicher Verwaltungstraditionen: erstens zwei kontinental-europäische, eine mit napoleonischer Tradition mit einer Fallstudie zu Frankreich (Philippe Bezes / Patrick Le Lidec), eine mit rö- 
misch-germanischer Tradition mit Fallstudien zu Deutschland (Julia Fleischer) und Italien (Francesco Stolfi); eine anglo-amerikanische Gruppe mit Fallstudien zu Australien (John Halligan), den USA (B. Guy Peters), Kanada (Donald Savoie) und dem Vereinigten Königreich (Martin Smith); sowie eine skandinavische Gruppe mit Beiträgen zu Schweden (Carl Dahlström / Jon Pierre), Dänemark (Lotte Jensen) und Finnland (Sirpa Kekkonen / Tapio Raunio). Abgeschlossen wird auch dieser Band mit einer Zusammenfassung der Ergebnisse sowie einer Herausarbeitung generalisierbarer Themen (Dablström / Peters / Pierre).

Insgesamt verdeutlichen die Studien sowie die Sammlung als Ganzes, dass ein Großteil der Herausforderungen und der Spannungsfelder (zum Beispiel zwischen zentripetalen und zentrifugalen Kräften) mit Prozessen der weiteren Evolution der Staatlichkeit über Einzelfälle hinaus einhergehen. Sie legen jedoch auch nahe, dass die Varianz in der Intensität der (Rück)Entwicklungen - zum Beispiel in der Ausprägung der Rezentrierung - mit dem jeweiligen Systemkontext zusammenhängt. Bei den Ländern, die am meisten von der Politisierung der Kernexekutive und Vorkehrungen zur verstärkten zentralen Exekutivsteuerung gekennzeichnet sind, handelt es sich beispielsweise eher um jene Systeme (gerade die angloamerikanische Gruppe), die vorher die stärkste Dezentrierung aufwiesen. Damit stellt Rezentrierung häufig eine Reaktion auf frühere Entwicklungen dar. Allerdings lassen sich aus diesen Studien keine zuverlässigen Aussagen über die Qualität des Regierens treffen. Dementsprechend kann beispielsweise von einem starken Exekutivzentrum mit weitreichender Kontroll- und Steuerungskapazität nicht zwangsläufig erwartet werden, dass dieses längerfristig orientierte oder ansonsten „bessere“ Politik verfolgt.

Der Themenkomplex von Regieren und Politik im Zeichen wachsender Komplexität sowie grenzen- und sektorenübergreifender Verzahnung kann daher durchaus als gemeinsamer Ausgangspunkt der vorliegenden Studien bezeichnet werden. Vor allem zeigt sich, dass Veränderungen der Gewaltenteilung, der Kompetenzverteilung und der Kontrollbeziehungen in horizontaler wie vertikaler Dimension politischer Systeme verlaufen. Dieser Wandel lässt sich jedoch keineswegs mit einer Verschiebung hin zur Regierungsunfähigkeit gleichsetzen. Dafür machen diese Bände allzu deutlich, dass politische Systeme - ob herkömmliche, sich entwickelnde oder neuerartige Gemeinwesen (wie die EU) - über bemerkenswerte Dynamik und Anpassungsvermögen verfügen. Konstatiert wird jedoch auch, dass etwa die Rückgewinnung von Entscheidungs- und Steuerungsfähigkeit nicht selten geminderte Ergebnisqualität nach sich zieht und bei bestimmten Tendenzen und Problematiken - wie Entscheidungsverflechtung, Verantwortungsdiffusion oder gar Entparlamentarisierung - zu einer Verstärkung beziehungsweise Verschärfung führen kann. Überhaupt stellen sie allesamt verdienstvolle Leistungen dar, auch dank der offensichtlichen Sorgfalt und analytischer Verdichtung der Herausgeber, die für ihre Bände anspruchsvolle - und konsistent eingehaltene - Analyserahmen sowie aussagekräftige Resümees erstellt haben. Überdies erbringen alle drei Bücher nicht nur eine fruchtbare Ergänzung des Forschungsstands zu Governance, Politik und Staatlichkeit im Wandel. Auch für die akademische Lehre eignen sie sich - ob vereinzelt oder im Dreiklang - als wertvolle Lektüre, die eine Vielfalt an Themen bei gleichzeitigen Überschneidungen bietet. Dies leisten sie nicht zuletzt - bei aller Komplexität der Gegenstände - mit beachtlicher Verständlichkeit. Sie liefern analytisch gehaltvolle Ansätze und plausible Antworten. Aber ein Verdienst dieser Beiträge ist noch wichtiger: Sie lassen keinen Zweifel an der nachhaltigen Bedeutung ihrer Fragen. 